\title{
Factors Influencing Surveillance for Hepatocellular Carcinoma in Patients with Liver Cirrhosis
}

\author{
Hager A. Ahmed Mohammed ${ }^{a} \quad J u$ Dong Yang a Nasra H. Giama ${ }^{a}$ \\ Jonggi Choi ${ }^{a}$ Hawa M. Ali ${ }^{a}$ Kristin C. Mara ${ }^{b}$ William S. Harmsen ${ }^{b}$ \\ Russell H. Wiesner ${ }^{a}$ Michael D. Leise ${ }^{a}$ Terry M. Therneau ${ }^{b}$ \\ Lewis R. Roberts ${ }^{a}$ \\ ${ }^{a}$ Division of Gastroenterology and Hepatology, Department of Medicine, and ${ }^{b}$ Division of \\ Biomedical Statistics and Informatics, Department of Health Sciences Research, Mayo Clinic, \\ Rochester, Minn., USA
}

\author{
Key Words \\ Cirrhosis $\cdot$ Hepatocellular carcinoma $\cdot$ Liver cancer $\cdot$ Surveillance/screening
}

\begin{abstract}
Objective: Hepatocellular carcinoma (HCC) is the second most common cause of cancer-related mortality worldwide, and a rising cause of cancer mortality in the U.S. Liver cirrhosis is the major risk factor for HCC. Surveillance of persons with cirrhosis facilitates early detection and improves outcomes. We assessed the surveillance rate for HCC within a major academic health system and identified factors influencing surveillance. Patients and Methods: We examined the surveillance rate for HCC using liver ultrasound, CT, or MRI, and factors influencing surveillance in a cohort of 369 Minnesota residents with cirrhosis seen at the Mayo Clinic between 2007 and 2009. Results: Ninety-three percent of cirrhosis patients received at least one surveillance study, but only $14 \%$ received the recommended uninterrupted semiannual surveillance. Thirty percent received $\geq 75 \%$ of recommended surveillance, and $59 \%$ received $\geq 50 \%$ of recommended surveillance. Factors increasing surveillance included gastroenterology or hepatology specialist visits $(p<0.0001)$, advanced liver disease as assessed by hepatic encephalopathy $(p=0.0008)$, and comorbid illness as assessed by diabetes mellitus $(p=$ 0.02). Age, sex, race, residence, cirrhosis etiology, or number of primary care visits did not significantly affect the rate of surveillance. Conclusions: While the rate of surveillance in a major academic health system was higher than reported in other studies, surveillance was heavily dependent on visits to a subspecialist. This suggests a major and urgent national need to improve identification of individuals at risk for HCC in the primary care setting and the initiation and maintenance of surveillance by primary care practitioners.
\end{abstract}




\section{Liver Cancer}

\section{Introduction}

Hepatocellular carcinoma (HCC) is the second most common cause of death from cancer worldwide and the ninth most common cause of death from cancer in the U.S. Cirrhosis is the major risk factor for HCC. The incidence of HCC in patients with cirrhosis is 2-7\% per year [1]. Due to increased prevalences of chronic hepatitis C (HCV) and nonalcoholic fatty liver disease (NAFLD), HCC incidence and mortality in the U.S. have increased markedly over the past four decades [2-5]. Similar epidemiologic trends are observed worldwide, particularly in China [6].

Because the majority of HCCs in the U.S. occur in individuals who are not under surveillance [7], most HCCs are diagnosed at intermediate to advanced stages, when curative treatment is no longer feasible. Consequently, HCC is the major cause of death among cirrhotic patients.

The Global HCC BRIDGE study of incidence and outcomes of HCC showed that in countries with a comprehensive program for risk identification and surveillance for HCC, namely Japan and Taiwan, approximately $70 \%$ of HCCs were detected at very early or early stages. Consequently, the 5-year survival of patients seen with HCC in both countries was 50-70\% [5]. In contrast, in other Asian countries such as China and Korea, and also in Europe and North America, only $30-40 \%$ of HCC cases were detected at very early or early stages, resulting in 5 -year survival rates of only $20-35 \%$. This strongly suggests that enhanced surveillance is associated with earlier diagnosis and improved prognosis of HCC. In randomized controlled trials, surveillance was also shown to enhance detection of early-stage HCC, increasing eligibility for curative treatment and improving survival $[8,9]$.

The American Association for the Study of Liver Diseases (AASLD) recommends semiannual surveillance for high-risk groups, including chronic hepatitis B (HBV) patients without cirrhosis beginning at the age of 40 for Asian-born males, 50 for Asian-born females, and 20 for African-born individuals, and all patients with cirrhosis [10]. The recommended screening test is ultrasonography (US), with the addition of AFP when US is not reliable due to obesity or operator inexperience.

HCC surveillance is underutilized in the U.S., covering fewer than $30 \%$ of patients at risk of HCC [10]. This underutilization contributes to the difficulty in assessing the effectiveness of surveillance in improving survival of HCC patients. The reasons underlying surveillance underutilization remain incompletely understood and must be appreciated and addressed in order to reduce the increasing burden of morbidity and mortality from HCC in the U.S.

In this study, we assessed the rate of HCC surveillance by imaging in patients with cirrhosis seen at the Mayo Clinic, Rochester, Minn., and the surrounding Mayo Clinic Health System and the factors influencing surveillance. We hypothesized that surveillance is underutilized and that surveillance is affected by subject demographics, severity of liver disease, presence of comorbid illness, and physician specialty.

\section{Methods}

This study was approved by the Institutional Review Board of the Mayo Clinic, Rochester, Minn., USA

\section{Study Population}

We conducted a retrospective cohort study including all Minnesota residents with cirrhosis who were seen at the Mayo Clinic between January 1, 2007 and December 31, 2009. Subjects were identified by querying the Mayo Clinic Life Sciences System database containing the electronic medical records of all patients seen at the Mayo Clinic. Cirrhosis cases were identified using codes 571.2, 571.5, and 571.6 from the International Classification of Diseases, Ninth Revision, Clinical Modification (ICD9-CM), which were assigned by specialized 


\section{Liver Cancer}

\begin{tabular}{l|l}
\hline Liver Cancer 2017;6:126-136 \\
\hline DOI: 10.1159/000450833 & $\begin{array}{l}\text { ( ) 2016 S. Karger AG, Basel } \\
\text { www.karger.com/lic }\end{array}$ \\
\hline
\end{tabular}

Ahmed Mohammed et al.: Factors Influencing Surveillance for Hepatocellular

Carcinoma in Patients with Liver Cirrhosis

coders. The diagnosis of cirrhosis was further confirmed by review of physicians' diagnoses as well as by corroborative evidence from histopathology, imaging (cirrhotic liver configuration, signs of portal hypertension) or platelet count $<120 \times 10^{9} / \mathrm{l}$ in the setting of chronic liver disease. Underlying chronic liver diseases were abstracted, including chronic HCV or HBV infection, alcoholic liver disease, NAFLD, primary or secondary biliary cirrhosis, primary sclerosing cholangitis, and other metabolic or genetic chronic liver diseases. Baseline demographic, clinical and laboratory data were obtained at the time of cirrhosis diagnosis.

Underlying etiologies of cirrhosis were categorized as: (1) alcoholic cirrhosis, defined by history of alcohol abuse, dependence, or average alcohol intake of more than $30 \mathrm{~g}$ daily for men and $16 \mathrm{~g}$ daily for women [11, 12], (2) NAFLD, defined by steatosis in liver images without a history of significant alcohol use or cryptogenic cirrhosis in patients with metabolic syndrome [13], (3) viral etiology including chronic HCV, defined by detectable HCV RNA or positive anti-HCV antibody with documented chronic liver disease, and chronic HBV, defined by positive serum HBsAg, and (4) other. The presence of ascites or encephalopathy, used as indicators of more severe liver disease, was ascertained by chart review.

Diabetes mellitus (DM) was assessed as a common comorbid illness generally associated with an increased number of health care visits. It was defined as follows: (1) physician diagnosis of DM, (2) use of insulin or oral antidiabetic agents, or (3) fasting glucose $\geq 126 \mathrm{mg} / \mathrm{dl}$ or $\mathrm{HbA}_{1 \mathrm{c}} \geq 6.5 \%$ on two separate occasions.

HCC diagnosis was confirmed by histopathological or radiologic criteria defined by new liver mass $\geq 1$ $\mathrm{cm}$ with characteristic features including arterial enhancement and delayed washout conforming to AASLD guidelines [10]. HCC stage was assessed using the Barcelona Clinic Liver Cancer (BCLC) staging system.

The specialist seen, gastroenterologist or hepatologist (GIH) versus internal medicine or family medicine primary care practitioner (PCP), was obtained from the Mayo Clinic databases.

Laboratory test results (platelet count, bilirubin, albumin and creatinine) were obtained from the Mayo Clinic databases. The Child-Pugh score was available in 323 (88\%) and the Model for End-Stage Liver Disease (MELD) score in 339 (92\%) subjects.

Abdominal imaging studies (US, CT, and MRI) performed at the Mayo Clinic, Rochester, Minn., and Mayo Clinic Health System Hospitals or Clinics were retrieved electronically. The results of abdominal imaging studies performed elsewhere and uploaded into the Mayo Clinic electronic medical records were abstracted via chart review. Imaging studies not relevant to HCC surveillance, such as coronary angiography, were excluded.

\section{Calculation of Surveillance Rate}

Assessment of surveillance started 3 months after the date of cirrhosis diagnosis or confirmation at the Mayo Clinic, and ended at the date of death, HCC diagnosis, liver transplant, or last follow-up (through December 31, 2013). The surveillance period was subdivided into intervals around the recommended interval of semiannual surveillance, with a 3-month window before and after, e.g. $6 \pm 3$ months, etc. To prevent overestimation of surveillance rates, only one abdominal imaging study was considered relevant during each target surveillance interval.

The surveillance rate was calculated by dividing the sum of the actual number of images performed by the sum of the expected number of images in the defined surveillance period for each subject. The surveillance rate was treated as a continuous variable in the univariate and multivariable analyses.

Patients with less than 6 months of follow-up were excluded. Patients diagnosed with hepatobiliary cancers or who underwent liver transplant within 6 months of cirrhosis diagnosis were also excluded (fig. 1). In a supplemental analysis with the goal of obtaining worst case estimates for surveillance rates, we also performed an analysis assuming that patients with less than 6 months of follow-up had dropped out from surveillance.

\section{Statistical Analysis}

Data are presented as median (25th-75th percentile) for continuous variables and percentages for categorical variables. To identify factors associated with surveillance, univariate analysis was performed for: age, sex, race, residence, etiology of cirrhosis (alcoholic cirrhosis, NAFLD, and viral etiology including HBV and HCV), severity of cirrhosis (ascites and/or encephalopathy), presence of comorbid DM, and average number of visits per year to different primary care or specialty clinics (internal medicine, family medicine, or GIH). A multivariable linear regression model was constructed to identify independent predictors of surveillance. The model was built via backward elimination using a cutoff $p$ value $\leq 0.1$. All analyses were two-tailed; a p value of $<0.05$ was considered significant. All statistical analyses used SAS ${ }^{\circledR}$ software (Cary, N.C., USA). 
Fig. 1. Study profile. $\mathrm{CCA}=$ Chol-

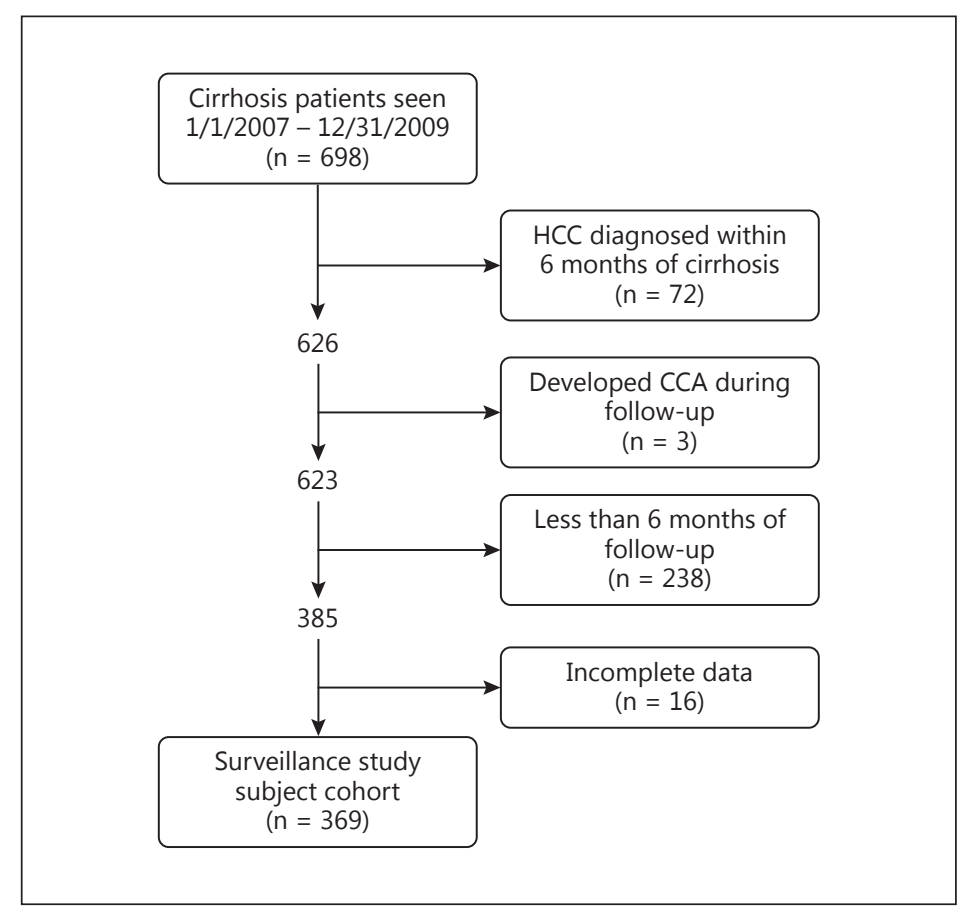
angiocarcinoma.

\section{Results}

\section{Patient Characteristics}

Between January 2007 and December 2009, 698 Minnesota residents with liver cirrhosis were seen at the Mayo Clinic, Rochester, Minn., USA. We excluded 238 (34\%) subjects with less than 6 months of follow-up, $72(10 \%)$ in whom HCC was diagnosed within 6 months of cirrhosis diagnosis, $3(0.4 \%)$ who developed cholangiocarcinoma during follow-up, and 16 (2\%) with incomplete data. A total of 369 subjects met the inclusion criteria (fig. 1).

The median age of patients with cirrhosis was 58 years $(25$ th -75 th percentile $=50-67$ ), $57 \%$ were men and $91 \%$ were white. DM was present in 33\%. The most common causes of cirrhosis were alcohol (32\%), NAFLD (23\%), and HCV (21\%). Ascites and encephalopathy were present in 46 and 18\% of subjects, respectively. The median Child-Pugh score was 8 (7-9) and $76 \%$ of subjects were Child-Pugh class B at the time of cirrhosis diagnosis. The median MELD score was 11 (8-14). Twenty-four (7\%) of the subjects developed HCC during a median follow-up of 48 months (table 1 ).

\section{Rates of Surveillance for HCC in Patients with Cirrhosis}

The total number of abdominal images performed at the Mayo Clinic or within the Mayo Clinic Health System, and outside images either uploaded to Mayo Clinic systems or scanned into the electronic medical record for the 369 subjects between January 2007 and December 2013 was 2,562 images, of which 1,363 were used to calculate the surveillance rate (some studies were outside the surveillance period, and the others were not counted because we counted only one image per 6 months). The absolute and cumulative percentages of patients receiving different levels of surveillance are shown in figure 2 and online supplementary table 1 (for all online suppl. material, see www.karger.com/doi/10.1159/000450833). Overall, $14 \%$ of the subjects received $100 \%$ semiannual surveillance by imaging studies throughout the eligibility period, $16 \%$ of subjects received $75-99 \%$ surveillance, $29 \%$ of 


\section{Liver \\ Cancer}

Table 1. Baseline characteristics of Minnesota residents with liver cirrhosis seen at the Mayo Clinic between January 2007 and December 2009
Fig. 2. Surveillance rates for Minnesota residents with liver cirrhosis seen at the Mayo Clinic 2007-2009.

\begin{tabular}{l|l}
\hline Liver Cancer 2017;6:126-136 \\
\hline DOI: 10.1159/000450833 & $\begin{array}{l}\text { @ 2016 S. Karger AG, Basel } \\
\text { www.karger.com/lic }\end{array}$ \\
\hline
\end{tabular}

Ahmed Mohammed et al.: Factors Influencing Surveillance for Hepatocellular Carcinoma in Patients with Liver Cirrhosis

\begin{tabular}{lc}
\hline Characteristic & Total $(\mathrm{n}=369)$ \\
\hline Age, years & $58(50,67)$ \\
Male & $209(57 \%)$ \\
Race & \\
$\quad$ White & $332(91 \%)$ \\
$\quad$ Black or African-American & $10(3 \%)$ \\
Other & $23(6 \%)$ \\
Diabetes & $121(33 \%)$ \\
Ascites & $169(46 \%)$ \\
Encephalopathy & $65(18 \%)$ \\
Etiology of cirrhosis & $117(32 \%)$ \\
$\quad$ Alcoholic cirrhosis & $83(23 \%)$ \\
NAFLD & $79(21 \%)$ \\
Chronic HCV & $8(2 \%)$ \\
Chronic HBV & $82(22 \%)$ \\
Other & $11(8,14)$ \\
MELD score & \\
Child-Pugh class & $32(10 \%)$ \\
A & $246(76 \%)$ \\
B & $45(14 \%)$ \\
C & $24(7 \%)$ \\
HCC diagnosed & $8(3,15)$ \\
GIH visits & $2.3(1.0,4.5)$ \\
GIH visits per year & $0.5(0.3,0.8)$ \\
Surveillance rate & $4(2,5)$ \\
Surveillance length, years & \\
\hline
\end{tabular}

Age and lab values are displayed as median (25th percentile, 75th percentile). Race: missing for 4 subjects. MELD: missing for 30 subjects. Child-Pugh: missing for 46 subjects.

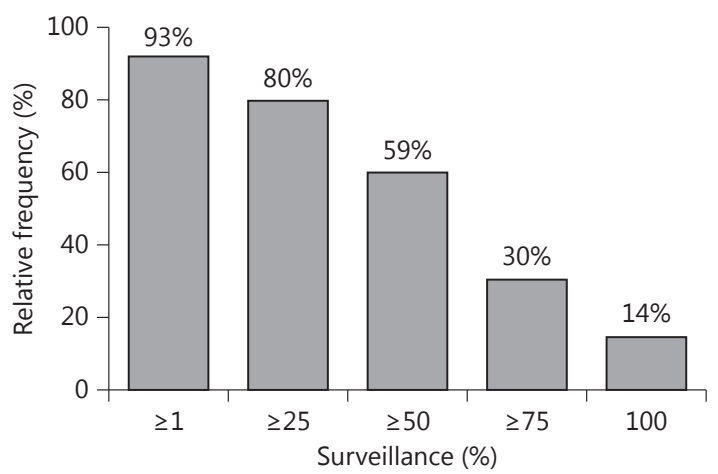

subjects received $50-74 \%$ surveillance, $21 \%$ of subjects received $25-49 \%$ surveillance, $13 \%$ of subjects received 1-24\% surveillance, and $7 \%$ of subjects received $0 \%$ surveillance. Expressed as cumulative surveillance, $30 \%$ of subjects received at least $75 \%$ of recommended surveillance (approximately 3 imaging studies per 24 months), and 59\% received at least $50 \%$ of the recommended surveillance (approximately 1 image per 12 months). At least one imaging study during the surveillance period (any imaging) was provided to $93 \%$ of the 
Table 2. Factors influencing surveillance, univariate analysis using surveillance rate as continuous variable

\begin{tabular}{|c|c|c|c|c|}
\hline Model & Variable & Regression coefficient & Standard error & $\mathrm{p}$ value \\
\hline 1 & Age & -0.002 & 0.001 & 0.10 \\
\hline \multirow[t]{3}{*}{2} & Gender & & & \\
\hline & Male & -0.023 & 0.033 & 0.48 \\
\hline & Female & 0.00 & & \\
\hline \multirow[t]{3}{*}{3} & Race & & & \\
\hline & White & -0.112 & 0.053 & 0.04 \\
\hline & Other & 0.00 & & \\
\hline 4 & Diabetes & 0.054 & 0.034 & 0.11 \\
\hline 5 & Ascites & 0.082 & 0.032 & 0.01 \\
\hline 6 & Encephalopathy & 0.126 & 0.042 & 0.003 \\
\hline \multirow[t]{5}{*}{7} & Etiology & & & 0.81 \\
\hline & Alcohol & -0.416 & 0.045 & $(0.35)$ \\
\hline & Viral etiology & -0.016 & 0.048 & $(0.73)$ \\
\hline & NAFLD & -0.031 & 0.048 & $(0.52)$ \\
\hline & Other & 0.00 & & \\
\hline 8 & Rochester residence & 0.012 & 0.045 & 0.78 \\
\hline 9 & Olmsted County residence & -0.006 & 0.042 & 0.88 \\
\hline 10 & Olmsted or adjacent county residence & 0.001 & 0.034 & 0.97 \\
\hline 11 & Internal medicine visits per year & 0.004 & 0.003 & 0.15 \\
\hline 12 & Family medicine visits per year & -0.002 & 0.003 & 0.55 \\
\hline 13 & GIH visits per year & 0.025 & 0.003 & $<0.0001$ \\
\hline
\end{tabular}

subjects, while $7 \%$ did not receive any imaging over the entire surveillance period [median follow-up 48 months (27-62)].

In order to address the possibility that the requirement of at least 6 months of follow-up after diagnosis of cirrhosis led to overestimation of surveillance rates, as individuals who did not receive at least 6 months of follow-up could have dropped out of surveillance, we repeated the analysis assuming that individuals excluded from the primary analysis due to having less than 6 months of follow-up did drop out of surveillance, thus creating worst case estimates for surveillance (online suppl. table 2). As expected, the surveillance rate estimates were lower, and reiterate the need for improved surveillance, with only $9 \%$ of subjects receiving $100 \%$ surveillance, $19 \%$ receiving at least $75 \%$ surveillance, and $37 \%$ of individuals receiving at least $50 \%$ of recommended surveillance.

\section{Determinants of HCC Surveillance}

Univariate analysis showed positive associations between HCC surveillance and the number of visits to a GIH per year ( $p<0.0001)$, presence of hepatic encephalopathy ( $p=$ $0.003)$, presence of ascites $(p=0.01)$, and non-white race $(p=0.04)$ (table 2). Age, sex, residence, etiology of cirrhosis, or number of PCP visits per year did not show significant associations with surveillance. By multivariable analysis, the number of GIH visits $(p<0.0001)$, and hepatic encephalopathy ( $\mathrm{p}=0.0008$ ) remained significant (table 3 ). The number of GIH visits was significantly associated with surveillance when analyzed with or without including liver transplant evaluation visits. Interestingly, DM was also significantly associated with receipt of surveillance by multivariable analysis $(p=0.02)$.

We further investigated the reasons for lack of surveillance in 26 of the 369 subjects who had no surveillance (median follow-up 36.6 months (11.9-51.3). There were four categories of reasons: for 13 patients (50\%), a recommendation for surveillance was made by the GIH, but either not implemented by the PCP or patients did not comply with the recommendation; 


\section{Liver Cancer}

Table 3. Factors influencing surveillance, multivariable linear regression using $\mathrm{p}$ value $\leq 0.1$

\begin{tabular}{l|l}
\hline Liver Cancer 2017;6:126-136 \\
\hline DOI: 10.1159/000450833 & $\begin{array}{l}\text { @ 2016 S. Karger AG, Basel } \\
\text { www.karger.com/lic }\end{array}$ \\
\hline
\end{tabular}

Ahmed Mohammed et al.: Factors Influencing Surveillance for Hepatocellular Carcinoma in Patients with Liver Cirrhosis

\begin{tabular}{lccc}
\hline Variable & $\begin{array}{l}\text { Regression } \\
\text { coefficient }\end{array}$ & $\begin{array}{l}\text { Standard } \\
\text { error }\end{array}$ & p value \\
\hline Race & & & \\
$\quad$ White & -0.084 & 0.049 & 0.088 \\
$\quad$ Other & 0.00 & & \\
Age & -0.002 & 0.001 & 0.094 \\
Diabetes & 0.075 & 0.032 & $0.020^{*}$ \\
Encephalopathy & 0.131 & 0.039 & $0.001^{*}$ \\
GIH visits per year & 0.025 & 0.003 & $<0.0001^{*}$ \\
\hline
\end{tabular}

Table 4. Factors influencing GIH visits

\begin{tabular}{lcccc}
\hline Term & Estimate & Standard error & t ratio & Prob $>|t|$ \\
\hline Age & -0.02 & 0.02 & -1.11 & 0.27 \\
White race & -1.10 & 0.78 & -1.41 & 0.16 \\
Diabetes & -0.81 & 0.50 & -1.63 & 0.10 \\
Ascites & 0.39 & 0.47 & 0.83 & 0.41 \\
Encephalopathy & -0.30 & 0.61 & -0.48 & 0.63 \\
Rochester & -0.07 & 0.65 & -0.1 & 0.92 \\
Olmsted and 6 surrounding counties & -0.32 & 0.49 & -0.65 & 0.52 \\
Olmsted County & -0.27 & 0.61 & -0.44 & 0.66 \\
Child-Pugh score & 0.15 & 0.18 & 0.83 & 0.41 \\
MELD & -0.03 & 0.04 & -0.59 & 0.55 \\
Etiology of cirrhosis (NAFLD = reference) & & & & $0.02^{*}$ \\
$\quad$ HCV & 1.85 & 0.70 & 2.64 & $0.01^{*}$ \\
$\quad$ Alcohol & 0.50 & 0.64 & 0.79 & 0.43 \\
$\quad$ Other than alcohol, NAFLD, or HCV & 1.54 & 0.68 & 2.28 & $0.02^{*}$ \\
\hline
\end{tabular}

$* \mathrm{p}<0.05$ (significant).

for 7 patients (27\%), there was failure in the transition of care between an inpatient diagnosis of cirrhosis and outpatient implementation of surveillance; for 4 patients (15\%), the GIH made the diagnosis of cirrhosis, but did not explicitly recommend surveillance; and for 2 patients (8\%), the diagnosis of cirrhosis was made by a radiologist or pathologist but not recorded in the ordering physician's clinical note, suggesting that it might have been overlooked.

\section{Predictors of GIH Visits}

We conducted univariate linear regression to assess predictors of GIH visits (table 4). The only significant predictor was the underlying etiology of cirrhosis $(p=0.02)$. Patients with HCV-induced cirrhosis had an average of 1.85 more GIH visits per year than those who had NAFLD ( $p=0.01$ ). Those who had an etiology other than alcohol, NAFLD, or HCV also had significantly more GIH visits per year (on average 1.54 more visits per year) than those with NAFLD ( $p=0.02)$. There was no significant difference in the number of GIH visits per year between those with alcohol-induced versus those with NAFLD-induced cirrhosis $(p=0.43)$. Thus HCV infection, which perhaps was considered to require specialty consultation for management, was more likely to be associated with GIH visits than alcoholic or fatty liver disease. Residential distance from the main tertiary medical center in Rochester, Minn., USA was not associated with a significant decline in GIH visits. 


\section{Liver

Ahmed Mohammed et al.: Factors Influencing Surveillance for Hepatocellular

Carcinoma in Patients with Liver Cirrhosis

\section{Impact of Surveillance on HCC Stage at Diagnosis}

To determine the impact of surveillance on HCC stage at diagnosis, and consequently potentially on patient outcomes, we examined whether surveillance was associated with earlier HCC stage at the time of diagnosis. Twenty-four HCCs were diagnosed during the study period. Ten (42\%) were BCLC stage A at diagnosis, $6(25 \%)$ were BCLC B, 3 (13\%) were BCLC C, and 5 (21\%) were BCLC D. We assessed whether more frequent surveillance (defined as $\geq 75 \%$ surveillance) was associated with early BCLC stage A HCC compared to intermediate, advanced or terminal BCLC stage (stages B, C and D grouped together). Of the 24 HCC cases, 14 (58\%) received $\geq 75 \%$ surveillance, of whom 7 (50\%) were diagnosed with early-stage disease (BCLC A) and 7 (50\%) were diagnosed with intermediate- to terminalstage disease (BCLC B-D). In comparison, $10(42 \%)$ received $<75 \%$ surveillance, of whom 3 $(30 \%)$ were diagnosed with early-stage disease (BCLC A) and 7 (70\%) were diagnosed with intermediate- to terminal-stage disease (BCLC B-D). Patients who received at least 75\% surveillance were 2.33 times more likely to be diagnosed with early BCLC stage A than those who did not. However, the difference was not statistically significant and had a wide $95 \%$ confidence interval, most likely due to the small number of cases [p value 0.33 (95\% confidence interval, 0.42-12.91), Fisher's exact test]. Thus, increased surveillance was associated with early-stage diagnosis, albeit not at a level of statistical significance due to the small numbers.

\section{Discussion}

In this study, we evaluated the rate of HCC surveillance provided to patients with cirrhosis at a major referral center and the surrounding health system and the determinants of surveillance. Surveillance by imaging was performed at least once on the majority of the patients in this study. However, regular semiannual surveillance was underutilized with a rate of consistent uninterrupted surveillance of only $14 \%$. Factors associated with increased surveillance utilization were the number of visits to a GIH per year, decompensated cirrhosis as evidenced by hepatic encephalopathy, and DM. Age, race, sex, residence, or etiology of cirrhosis did not significantly impact the receipt of HCC surveillance.

AASLD recommends semiannual HCC surveillance for patients with cirrhosis, with the goal of early detection of HCC and improved patient outcomes. However, surveillance is underutilized in the U.S. A meta-analysis of nine studies showed a pooled HCC surveillance rate of $18.4 \%$ [14]. Most of these surveillance studies used a less stringent definition of annual rather than semiannual surveillance. A recent study assessed the rate of semiannual surveillance and reported that only $1.7 \%$ of eligible patients received semiannual surveillance, while $13 \%$ received annual surveillance [15]. Our study was unique in both using a gold standard of semiannual surveillance and calculating surveillance rate as a continuous variable to enhance the power of the analysis. We expended significant effort to capture all images performed on our study population at the major Mayo Clinic referral center as well as in the surrounding Mayo Clinic Health System. Compared with other studies, we found that the rates of semiannual (14\%) and annual (59\%) HCC surveillance were substantially higher than reported in other U.S. studies; however, in comparison to countries with national surveillance programs such as Taiwan and Japan, HCC surveillance was still significantly underutilized in our study population.

The determinants of HCC surveillance are potentially key drivers of the quality of clinical care and provide important insights into possible strategies for improving surveillance rates and maximizing the impact of surveillance on patient outcomes. As shown in previous studies, we found that the frequency of visits to GIH was a strong determinant of the likelihood of 
Ahmed Mohammed et al.: Factors Influencing Surveillance for Hepatocellular

Carcinoma in Patients with Liver Cirrhosis

surveillance, whereas increasing numbers of PCP visits did not improve the chances of receiving surveillance. A previous study using the Surveillance, Epidemiology, and EndResults (SEER) - Medicare databases reported that periodic HCC screening was 2.8 and 4.5 times more likely in patients who received GIH care with or without internal medicine or family physician visits compared to patients who did not receive subspecialty care [7]. Another study showed that the single most important predictor of semiannual surveillance was the number of hepatology visits [15]. A third study reported that HIV patients co-infected with HBV are 5.5 times more likely to receive HCC surveillance if they are seen by GIH compared to HIV providers ( $\mathrm{p}<0.001$ ) [16]. This is a critical factor from a health policy perspective, as the relatively sparse national distribution of hepatology specialists is such that if nationwide surveillance depends on patients seeing a hepatologist, it will be impossible to achieve the near universal surveillance needed to improve U.S. national survival outcomes for HCC to the levels achieved in countries such as Taiwan and Japan. Similar to the development of Project ECHO (Extension for Community Healthcare Outcomes), which has substantially improved HCV treatment rates and outcomes in communities with low access to hepatologists by partnership with primary care providers, there is an urgent need to pair the identification of those at risk for HCC with robust HCC surveillance using primary care providers as the main drivers of the surveillance algorithm, as is currently the case with colon, breast, cervix, and lung cancer surveillance in the U.S. [17].

Second, hepatic decompensation was significantly associated with increased surveillance. In our study, surveillance was most strongly associated with presence of hepatic encephalopathy, while in the study by Singal et al. [18], ascites was a stronger predictor of surveillance than hepatic encephalopathy. The study by Singal et al. [18] compared patients with early- versus advanced-stage presentation of HCC to determine the points of failure of the surveillance program, while our study approached the question by evaluating determinants of surveillance in persons with cirrhosis. The difference in study designs may in part account for the difference in association of hepatic encephalopathy and ascites with surveillance. Nevertheless, both results suggest that persons with more advanced cirrhosis, who may be less likely to have long-term benefit from surveillance for HCC, are preferentially targeted for surveillance, perhaps because their liver disease is more obvious. This suggests a need for improved identification of individuals with compensated cirrhosis who are likely to derive the greatest benefit from surveillance, early detection and treatment with curative intent. The tendency to survey individuals with more severe liver disease may also result in lower cost-effectiveness of surveillance strategies. Importantly, the low overall rates of surveillance suggest the need for concerted effort to initiate and continue surveillance in individuals identified to be at high risk of HCC.

To our knowledge, this study is the first study to assess the impact of DM as a comorbid disease on surveillance rates. We found that the presence of concomitant DM was associated with increased HCC surveillance among cirrhosis patients. This may be related to a higher rate of physician visits by diabetic patients compared to other patients, or alternatively to a higher likelihood of abnormal liver tests occurring due to NAFLD in diabetic patients. The reason for the association of DM with increased HCC surveillance is unclear. Further analysis did not reveal any associations of DM with features of advanced liver disease, including ascites, hepatic encephalopathy, MELD score, or Child-Pugh class.

Factors that were examined but not shown to be significantly associated with surveillance in the multivariate analysis included race, residence, and etiology of cirrhosis. Nonwhite race was significantly associated with increased surveillance by univariate analysis, but the association did not persist on multivariate analysis. The univariate association could be due to physician recognition of non-white race as indicating a high-risk population or to a false positive association as the study subjects were predominantly white (91\%). 


\section{Liver

Ahmed Mohammed et al.: Factors Influencing Surveillance for Hepatocellular

Carcinoma in Patients with Liver Cirrhosis

The reasons for lack of HCC surveillance identified in the 26 patients who had no surveillance included patients in whom a recommendation for surveillance was made by the GIH, but either not implemented by the PCP or patients did not comply with the recommendation; patients for whom there was failure in the transition of care between an inpatient diagnosis of cirrhosis and outpatient implementation of surveillance; patients for whom the GIH made the diagnosis of cirrhosis, but did not explicitly recommend surveillance; and patients for whom the diagnosis of cirrhosis was made by a radiologist or pathologist but not recorded in the ordering physician's clinical note, suggesting that it might have been overlooked. Unfortunately, the small number of patients with no surveillance precluded meaningful comparison of differences in the frequencies of these reasons.

Our study has a number of limitations. The first is its retrospective design, which limited availability of some variables due to missing data. Second, there is a possibility of referral bias, since the study is based at a tertiary medical center. To minimize referral bias, we restricted the study population to Minnesota residents with a minimum follow-up of 6 months, allowing us to select the population of patients who most likely obtained both their primary and specialty care at our institution and in the surrounding Mayo Clinic Health System sites. Additionally, we compared surveillance rates between individuals residing in Rochester and Olmsted County, immediately surrounding our institution, compared to residents of other cities and counties in Minnesota. There were no statistically significant differences in surveillance rates. A third limitation is that we were only able to review imaging studies performed at the Mayo Clinic or Mayo Clinic Health System Hospitals, images uploaded from outside hospitals, or imaging studies performed by non-Mayo Clinic providers for which the radiology reports had been received and scanned into the Mayo Clinic electronic record. Since surveillance rates for individuals resident in Rochester or surrounding Olmsted County were similar to the rates for individuals resident in other Minnesota cities and counties, it appears that for Minnesota residents included in our study, the distance from Rochester was not an impediment to surveillance. A fourth limitation is that provider intent of performing surveillance was not assessed, thus some studies might have been performed for other indications. This limitation was mitigated by only accepting one imaging study for every 6-month period of follow-up. Another limitation is that we could not assess the impact of socioeconomic status, insurance coverage, or level of educational attainment on surveillance rate.

Overall, the strengths of this study outweigh the limitations. Our findings of underutilization of surveillance and the significant association of specialty care with surveillance confirm and extend the results of previous studies. Most interestingly, we observed that sicker patients with hepatic decompensation and comorbidities such as DM are more likely to undergo surveillance. This may be due to the fact that patients with hepatic decompensation are more likely to be referred to a GIH and the increased likelihood of abnormal liver tests in individuals with diabetes. Paradoxically, this observation suggests that persons with compensated cirrhosis, who are the individuals most likely to benefit from surveillance, are actually less likely to receive surveillance because their liver disease is less obvious. This underscores the importance of training PCPs in the recognition of cirrhosis and the appreciation of cirrhosis as a major risk factor for HCC and liver-related death. It is also critically important to assess the knowledge and raise the awareness of the appropriate methods and frequency of HCC surveillance among PCPs. A potential durable solution to this issue may lie within systems engineering, whereby electronic medical records systems can be programmed to flag and notify providers and patients when they are due for HCC surveillance.

In conclusion, this retrospective cohort study demonstrates continued underutilization of HCC surveillance in the U.S. despite long-standing guideline recommendations. Surveillance is positively associated with number of visits to a GIH, but not with visits to a PCP. Since there is more limited distribution and access to GIH specialists, programs to enhance the 
Ahmed Mohammed et al.: Factors Influencing Surveillance for Hepatocellular

Carcinoma in Patients with Liver Cirrhosis

recognition of persons at risk for HCC and increase the knowledge and encourage the application of HCC surveillance by PCP should be key components of systematic efforts to reduce the burden of illness and death from HCC. Further, this study sheds light on determinants of surveillance in patients with cirrhosis. Patients with decompensated cirrhosis and comorbid illness (DM) are more likely to receive surveillance, while they are somewhat less likely to derive sustained benefit from surveillance.

\section{Acknowledgements}

Sources of funding: Mayo Clinic. Mayo Clinic Cancer Center (CA15083). Mayo Center for Clinical and Translational Sciences (NIH/NCRR CTSA Grant Number UL1 TR000135).

\section{Disclosure Statement}

Potential conflict of interest: Lewis R. Roberts receives grant funding from: Gilead Sciences; Wako Life Sciences and BTG International. All funds are provided to the institution with no personal compensation. The remaining authors disclosed no interest.

\section{References}

1 Baffy G, Brunt EM, Caldwell SH: Hepatocellular carcinoma in non-alcoholic fatty liver disease: an emerging menace. J Hepatol 2012;56:1384-1391.

2 Jemal A, Bray F, Center MM, Ferlay J, Ward E, Forman D: Global cancer statistics. CA Cancer J Clin 2011;61: 69-90.

3 Yang JD, Roberts LR: Hepatocellular carcinoma: a global view. Nat Rev Gastroenterol Hepatol 2010; 7:448458.

4 Hassan MM, Frome A, Patt YZ, El-Serag HB: Rising prevalence of hepatitis C virus infection among patients recently diagnosed with hepatocellular carcinoma in the United States. J Clin Gastroenterol 2002;35:266-269.

5 Park JW, Chen M, Colombo M, Roberts LR, Schwartz M, Chen PJ, Kudo M, et al: Global patterns of hepatocellular carcinoma management from diagnosis to death: the BRIDGE Study. Liver Int 2015;35:2155-2166.

6 International Agency for Research on Cancer: GLOBOCAN 2012: Estimated Cancer Incidence, Mortality and Prevalence Worldwide in 2012. IARC CancerBase No. 11, 2012.

7 Davila JA, Morgan RO, Richardson PA, Du XL, McGlynn KA, El-Serag HB: Use of surveillance for hepatocellular carcinoma among patients with cirrhosis in the United States. Hepatology 2010;52:132-141.

8 Hassett M, Yopp AC, Singal AG: Surveillance for hepatocellular carcinoma: how can we do better? Am J Med Sci 2013;346:308-313.

9 Zhang BH, Yang BH, Tang ZY: Randomized controlled trial of screening for hepatocellular carcinoma. J Cancer Res Clin Oncol 2004;130:417-422.

10 Bruix J, Sherman M: Management of hepatocellular carcinoma: an update. Hepatology 2011;53:1020-1022.

11 Donato F, Tagger A, Gelatti U, Parrinello G, Boffetta P, Albertini A, Decarli A, et al: Alcohol and hepatocellular carcinoma: the effect of lifetime intake and hepatitis virus infections in men and women. Am J Epidemiol 2002; 155:323-331.

12 O'Shea RS, Dasarathy S, McCullough AJ: Alcoholic liver disease. Hepatology 2010;51:307-328.

13 Marrero JA, Fontana RJ, Su GL, Conjeevaram HS, Emick DM, Lok AS: NAFLD may be a common underlying liver disease in patients with hepatocellular carcinoma in the United States. Hepatology 2002;36:1349-1354.

14 Singal AG, Yopp A, Skinner CS, Packer M, Lee WM, Tiro JA: Utilization of hepatocellular carcinoma surveillance among American patients: a systematic review. J Gen Intern Med 2012;27:861-867.

15 Singal AG, Li X, Tiro J, Kandunoori P, Adams-Huet B, Nehra MS, Yopp A: Racial, social, and clinical determinants of hepatocellular carcinoma surveillance. Am J Med 2015;128:90.e1-e7.

16 Hearn B, Chasan R, Bichoupan K, Suprun M, Bagiella E, Dieterich DT, Perumalswami P, et al: Low adherence of HIV providers to practice guidelines for hepatocellular carcinoma screening in HIV/hepatitis B coinfection. Clin Infect Dis 2015;61:1742-1748.

17 Arora S, Kalishman S, Thornton K, Dion D, Murata G, Deming P, Parish B, et al: Expanding access to hepatitis C virus treatment - Extension for Community Healthcare Outcomes (ECHO) project: disruptive innovation in specialty care. Hepatology 2010;52:1124-1133.

18 Singal AG, Marrero JA, Yopp A: Screening process failures for hepatocellular carcinoma. J Natl Compr Canc Netw 2014;12:375-382. 